RESEARCH REPORT

\title{
The burden of food related ill health in the UK
}

\author{
Mike Rayner, Peter Scarborough
}

See end of article for authors' affiliations

J Epidemiol Community Health 2005;59:1054-1057. doi: 10.1136/jech.2005.036491

.....................

Correspondence to: Mr P Scarborough, British

Heart Foundation Health Promotion Research Group, Department of Public Health, University of Oxford, OX3 7LF, UK; peter.scarborough@ dphpc.ox.ac.uk

Accepted for publication 8 August 2005

\begin{abstract}
Study objective: To quantify the burden of ill health in the UK that can be attributed to food (the burden of food related ill health).

Design: Review and further analysis of the results of work concerned with estimates of the burden of disease measured as morbidity, mortality, and in financial terms and with the proportion of that burden that can be attributed to food.

Main results: Food related ill health is responsible for about $10 \%$ of morbidity and mortality in the UK and costs the NHS about $£ 6$ billion annually.

Conclusions: The burden of food related ill health measured in terms of mortality and morbidity is similar to that attributable to smoking. The cost to the NHS is twice the amount attributable to car, train, and other accidents, and more than twice that attributable to smoking. The vast majority of the burden is attributable to unhealthy diets rather than to food borne diseases.
\end{abstract}

W hen devising food or health policy, policy makers make implicit assumptions about the burden of food related ill health. For example, if they think it is large then policy will be directed towards reducing the burden. If they think it is small then policy will be directed towards achieving other objectives. Information about the burden is not the only information needed when setting priorities. In addition policy makers need information about the effectiveness-particularly the cost effectiveness-of interventions. But we suggest that implicit assumptions about the burden of food related ill health should be made more explicit through quantification.

There are two main problems in quantifying the burden of ill health related to food and in particular when comparing the burden with that related to other causes: measurement and attribution.

There are basically three ways that the burden of ill health can be measured: mortality, morbidity, and money. There are several ways of measuring the mortality burden, for example, numbers of deaths, years of life lost in early death, etc. There are many ways of measuring the morbidity burden: numbers of incident/prevalent cases, days in hospital, days of certified incapacity, years of life lost in disability, etc. There is just one way in which the financial burden can be measured in any one country-the currency of that country-but various ways of coming to a conclusion as to the extent of the burden in that currency.

Different ways of measuring the burden lead to different assessments of its size. Measuring the burden is therefore not just a technical issue but also an ethical issue. Values come into play in choosing the particular aspect of the burden to measure, for example, just measuring numbers of deaths means a death at age 80 and a death at age 50 have the same significance. But practicalities also dictate the choice of measure. For health policy purposes it will normally be best to measure the burden of ill health in terms of mortality or morbidity. But mortality and morbidity cannot be used as measures of the burden in wider policy arenas: for example comparing the externalised costs-including the health costs-of different food policies (say different types of agricultural policies) can only be done in terms of money.

The second difficulty in quantifying burdens is attribution. Quantifying the extent to which ill health is related to particular causes is complex. Murray and Lopez distinguish between the attributable burden-"the difference between burden currently observed and burden that would have been observed under an alternative population distribution of exposure" —and the avoidable burden - "the reduction in the future burden...if the current levels of exposure to a risk factor were reduced to those specified by the counterfactual distribution of exposure" ${ }^{\prime 1}$ This distinction between attributable and avoidable burdens applies when examining burdens of ill health by disease as well as by cause. ${ }^{2}$ The implications of the distinction have, as yet, been barely explored in relation to policy. An understanding of the avoidable burden of disease or cause can only come from combining the results of studies of the effectiveness of interventions with information about the extent of the attributable burden.

In this paper we are concerned with the burden of food related ill health (or more precisely the burden of ill health that can be attributed to food). It is well established that many diseases are food related. ${ }^{3}$ Some diseases are caused by food borne pathogens, other diseases are caused by the over or under consumption of nutrients or other components of foods. The aim of this paper is to quantify the burden of food related ill health in the UK for two reasons: firstly to compare the size of the burden with that attributable to other causes and secondly to assess which aspects of the burden are more important.

\section{METHODS}

Measuring the burden of food related ill health in terms of mortality and morbidity is simpler than measuring it in financial terms. However as the debate about the work of the World Health Organisation's global burden of disease project ${ }^{5}$ shows, measuring burdens by any means is by no means either simple or uncontroversial. ${ }^{6}$ Nevertheless the results of this project constitute a possible starting point for an analysis of the burden of food related ill health.

Here we first identified the total burden of food related diseases using the results of the WHO global burden of disease project. Then we calculated the proportion of that

Abbreviations: PAF, population attributable fraction; CVD, cardiovascular disease; YLL, years of life lost; DALY, disability adjusted life years; YLD, years of life lost in disability; CHD, coronary heart disease 
Table 1 Amount of deaths (mortality), years of life lost in early death (YLLs), years of life lost in disability (YLDs), and disability adjusted life years (DALYs) in WHO EUR-A region, 2002

\begin{tabular}{lcccc}
\hline Cause & \% Of mortality & \% Of YLLs & \% Of YLDs & \% Of DALYs \\
\hline Infectious and parasitic diseases & 1.3 & 1.9 & 1.5 & 1.7 \\
$\quad$ Diarrhoeal diseases & 0.0 & 0.0 & 0.4 & 0.2 \\
Nutrient deficiencies & 0.2 & 0.1 & 1.0 & 0.6 \\
Cardiovascular disease & 41.1 & 29.5 & 6.4 & 17.1 \\
$\quad$ Coronary heart disease & 17.1 & 13.7 & 1.1 & 6.9 \\
$\quad$ Stroke & 10.6 & 6.9 & 3.6 & 5.1 \\
Diabetes & 2.4 & 1.9 & 2.4 & 2.1 \\
Cancer & 27.3 & 32.8 & 3.1 & 16.9 \\
Dental caries & 0.0 & 0.0 & 0.7 & 0.4 \\
Neuropsychiatric disorders & 4.7 & 4.8 & 45.3 & 26.5 \\
Injuries & 4.8 & 11.5 & 4.7 & 7.9 \\
Other & 18.2 & 17.5 & 34.9 & 26.8 \\
Total & 100.0 & 100.0 & 100.0 & 100.0 \\
\hline Source: WHO, 2003. & & & & \\
\hline
\end{tabular}

burden that can be attributed to food (the population attributable fraction (PAF)) using the results of the related comparative risk assessment project. ${ }^{78}$ We then sought to validate our results (triangulate them) with reference to other studies.

Quantifying the burden of food related ill health in financial terms is even more problematic. The cost to the health service of preventing and treating diseases is the simplest way of measuring burdens of ill health in financial terms. These costs are known as direct costs. It is also common to put a financial value to non-health service costs, for example, to employers in sickness pay, to unpaid carers for time spent in caring, to patients for lost earnings, etc. These costs are known as indirect costs. Cost of illness studies assessing the direct and indirect costs of different diseases are common but carried out using varying methods so their results cannot easily be compared.

The only study that has ever been carried out in the UK that has sought to assess the costs-and then only the direct costs-of different diseases on a comparable basis is a study published by the National Health Executive in 1996 describing results for the financial year 1992/1993. ${ }^{9}$ This study began with the budget for the NHS and sought to attribute costs to different International Classification of Disease (ICD) chapters. Here we used that study's results, together with our estimates of the proportions of ill health that are food related (PAFs), to make an estimate of the cost of food related ill health to the NHS. Again we then sought to triangulate our results.

\section{RESULTS}

\section{Mortality and morbidity}

As shown in table 1, the WHO's global burden of disease project $^{5}$ shows that in developed European countries cardiovascular disease (CVD) and cancer together constitute the majority-about $62 \%$ - of years of life lost in early death (YLLs) (and 68\% of total mortality), suggesting to health policy makers that these diseases should be the priority when it comes to devising health policy. Indeed CVD and cancer are priorities of national health policy in the UK and it is recognised that these diseases are diet related. ${ }^{10}$

But if the concern of policy is morbidity rather than mortality then the focus should be on diseases other than CVD and cancer-such as neuropsychiatric disorders. CVD and cancer only contribute $10 \%$ of years of life lost in disability (YLDs) whereas neuropsychiatric disorders contribute $45 \%$.

Measuring the burden in terms of both mortality and morbidity-that is, in disability adjusted life years (DALYs: the sum of YLLs and YLDs ${ }^{5}$ - - suggests that 34\% of the burden of ill health, is attributable to CVD and cancer: now more than neuropsychiatric disorders at $26 \%$.

The implicit assumption in the calculation of DALYs is that YLLs and YLDs are of equal significance, but note that most of the DALYs attributable to CVD and cancer are from years of life lost in early death rather than in disability.

Table 1 shows that just over $37 \%$ of DALYs are from food related diseases (highlighted in bold). It suggests, however, that food borne diseases-such as salmonella

Table 2 DALYs lost attributable to selected causes in the European Union and Australia, around 1995, and WHO EUR-A region, 2000

\begin{tabular}{llll}
\hline & EU (\%) & Australia (\%) & EUR-A region (\%) \\
\hline Smoking & 9.0 & 9.7 & 12.2 \\
Alcohol consumption & 8.4 & 2.1 & 6.6 \\
Diet (including overweight) & 8.3 & 9.6 & 15.5 \\
Overweight and obesity & 3.7 & 4.3 & 6.9 \\
Low fruit and vegetable intake & 3.5 & 2.7 & 2.3 \\
High saturated fat intake/low & 1.1 & 2.6 & 6.4 \\
polyunsaturated fat intake* & 1.4 & 6.7 & 2.8 \\
\hline Physical inactivity & & & 2.7 \\
\hline
\end{tabular}

*Technically the PAF for high blood cholesterol reported by the Australian study and the comparative risk Assessment project. Estimates for the PAFs for high blood pressure (although partly related to diet-particularly sodium intakes) have been omitted from this table to avoid duplication (for example, with overweight). Sources: National Institute of Public Health, Stockholm, 1997," Mathers et al, 1999'2 and comparative risk assessment project. $^{78}$ 
Table 3 Percentage of total NHS costs attributable to different diseases in 1992/1993 and 2001/2002

\begin{tabular}{|c|c|c|c|c|}
\hline & $\begin{array}{l}\% \text { Of total NHS costs in } \\
1992 / 1993^{*}\end{array}$ & Costs† ( $£$ billion) 2002 & Costs $\ddagger$ (£ billion) 2002 & Costs§ (£ billion) 2002/2003 \\
\hline Infectious diseases & 1 & 0.7 & & 0.8 \\
\hline Cardiovascular disease & 12 & 8.4 & & 5.8 \\
\hline $\begin{array}{l}\text { Coronary heart disease } \\
\text { Stroke }\end{array}$ & & & 2.4 & \\
\hline $\begin{array}{l}\text { Diabetes and other diseases of } \\
\text { the hormonal and immune } \\
\text { systems }\end{array}$ & 2 & 1.4 & 1.3 & 1.4 \\
\hline Cancer & 4 & 2.8 & 2.5 & 3.3 \\
\hline $\begin{array}{l}\text { Dental caries and other diseases } \\
\text { of the digestive system }\end{array}$ & 8 & 5.6 & & 2.3 \\
\hline $\begin{array}{l}\text { Neuropsychiatric disorders and } \\
\text { diseases of the nervous system }\end{array}$ & 25 & 17.6 & & 10.9 \\
\hline Injuries & 4 & 2.8 & & 3.3 \\
\hline Other & 45 & 31.6 & & 32.2 \\
\hline Total & 100 & 70.2 & & 60.0 \\
\hline
\end{tabular}

poisoning-constitute a comparatively minor burden compared with diet related diseases: about $0.2 \%$ of DALYs are attributable to food borne diseases (mainly diarrhoeal diseases).

Table 1 also shows that, apart from CVD, cancer, and diabetes, other diet related diseases such as dental caries and diseases attributable to nutritional deficiencies, do not constitute a major burden. CVD, cancer, and diabetes together are responsible for $36 \%$ of DALYs while other diet related diseases are only responsible for another $1 \%$.

Of course not all of the burden of CVD, cancer, and diabetes is directly attributable to food. All diseases have multiple causes. CVD—to take just one example-is caused by poor diets, smoking, physical inactivity, stress, etc. Only a proportion of the burden is attributable to food.

What then is this proportion? As yet only a rough estimate is possible. Probably the best estimate comes from examining the results of the WHO's comparative risk assessment project. ${ }^{78}$ This project has involved an analysis of the burden of disease that can be attributed to a range of risk factors (that is, the development of PAFs). One of the risk factors considered was low fruit and vegetable consumption, and three others were food related: overweight and obesity; high blood cholesterol levels, and high blood pressure levels but the project did not investigate the extent to which levels of these three risk factors are diet related so various assumptions have to be made in using the results.

Table 2 shows some of the results of this study together with the results of two other studies, one from Sweden ${ }^{11}$ and one from Australia, ${ }^{12}$ which estimate the burden in DALYs of certain risk factors for the European Union and Australia respectively. These three studies are the most methodologically robust calculations of the proportion of the burden of food related mortality and morbidity that we have at the present time. If we sum the effects (PAFs) for the individual diet related risk factors, the comparative risk assessment project shows that about $15 \%$ of all DALYs in developed economies are food related and the Swedish and Australian studies that that about $10 \%$ of all DALYs are food related.

It should be noted that for all three studies the combined effects of any group of risk factors will often be less than the sum of their separate effects as there are likely to be interactions between risk factors. Nevertheless, on a conservative basis it would seem that about $10 \%$ of DALYs are food related in developed countries such as the UK.

It is variously claimed that about one third of the mortality and morbidity attributable to $\mathrm{CVD}^{13}$ and one third of the mortality and morbidity attributable to cancer $^{14}{ }^{15}$ are food related. Both these estimates while widely used by policy makers are weak methodologically. For example, the estimate for cancer was originally calculated long before much of the evidence linking diets with cancer became available.

As CVD and cancer together constitute about 34\% of DALYs lost (table 1 ) and one third of $34 \%$ is $11 \%$, then this estimate accords well with the estimate derived from table 2 .

Table 2 also shows that all three studies indicate that about $10 \%$ of DALYs are attributable to smoking.

\section{ECONOMIC BURDEN}

Table 3 shows the \% of total NHS costs attributable to different diseases in 1992/1993 from the study published by the NHS Executive in $1996 .{ }^{\circ}$ It also shows the amounts of health care expenditure in the UK in 2002 attributable to these diseases if the proportions remained the same as in 1992/1993. There will, of course, have been significant differences in the absolute levels of health care costs for different diseases between 1992/1993 and 2002 but we think that differences in the proportions of the overall costs will have been less. As there is no more recent, good quality, study of the costs attributable to different diseases the 1996 study remains our best source of such estimates.

Table 3 also shows some direct costs of selected diseases taken from a recent report on the state of public health in the UK-the Wanless Report ${ }^{16}$ - and some recent figures produced by the Department of Health in England ${ }^{17}$-for the resources available to treat different conditions. Both of these sets of figures were published without explanation of how they were calculated.

Table 3 suggests that around $28 \%$ of NHS costs can be attributed to readily identifiable food related diseases (highlighted in bold) amounting to about $£ 18$ billion annually in

\section{What this paper adds}

This paper provides scientific rationale for the claim that food related ill health costs the NHS at least £6 billion each year. This updates a similar claim that was recently made in "Choosing a better diet: a food and health action plan", part of the delivery plan for the UK Department of Health's white paper "Choosing Health". 
2002. The table also shows that CVD and cancer are together responsible for most of that $£ 18$ billion.

It also shows that dental caries while responsible for only a small percentage of DALYs is responsible for a much larger proportion of health care costs. The budget for general dental services was $3.8 \%$ of the total budget in $2002 / 2003^{16}$ while table 1 suggests that dental caries is only responsible for $0.4 \%$ of lost DALYs.

Table 3 also shows that our estimates for the health care costs attributable to different diseases accord well with some recent calculations carried out in the course of preparing the Wanless Report. ${ }^{16}$ Note that although our estimate of $£ 8$ billion for the costs attributable to CVD is much higher than the $£ 2.4$ billion attributable to coronary heart disease (CHD) according to the Wanless Report-a recent study has found that CHD is responsible for only $24 \%$ of the costs attributable to $\mathrm{CVD} .{ }^{19}$ In contrast table 1 shows that CHD is responsible for $40 \%$ of the DALYs attributable to CVD.

Table 3 also shows that our estimates derived from using the results of the NHS Executive study are generally similar to the figures produced by the Department of Health. ${ }^{17}$

Assessing the proportion of the burden of ill health measured in financial terms that is food related is not simple because-as we have seen with dental caries and CHD-there is only some relation between the burden measured in $£$ or in DALYs. However, it might be reasonable to suggest that as about a third of DALYS are attributable to food related diseases and about $10 \%$ of all DALYs are food related then these proportions could also be applied to costs.

One third of the costs to the health service of the readily identifiable food related diseases shown in table 3 is $£ 6$ billion. This is twice the $£ 3$ billion cost of accidents including car (and indeed rail) accidents also shown in table 3 The $£ 6$ billion might also cautiously be compared with economic costs of food borne diseases recently calculated by Pretty and his colleagues. They argue that bovine spongiform encephalopathy (BSE) and variant Creutzfeldt-Jakob disease (vCJD) have recently had direct and indirect costs of $£ 0.6$ billion annually and that bacterial and viral outbreaks in food have cost $£ 0.2$ billion anually. ${ }^{20}$ Note that although these costs are substantial they are comparatively small compared with $£ 6$ billion from all food related diseases.

The $£ 6$ billion is also over three times one commonly quoted estimate ( $£ 1.5$ billion annually) for the cost of smoking to the $\mathrm{NHS}^{21}$ and far higher than recent official estimates for the cost of obesity of $£ 479$ million annually. ${ }^{22}$ This estimate for the cost of obesity was derived in a similar way to our estimate of the cost of food related ill health-that is, by applying PAFs to costs for a series of obesity related diseases. However, as the estimate was only concerned with obesity the range of diseases considered was smaller than ours (for example, dental caries was not considered at all and only colon, ovarian, prostate, endometrial, and rectal cancers).

\section{CONCLUSIONS}

That food related ill health is responsible for about $10 \%$ of DALYs lost in the UK and costs the NHS about $£ 6$ billion annually are obviously crude estimates. Nevertheless they are probably reasonable.

The estimates suggest that the burden of food related ill health is large, compared with say smoking, and suggest that food related ill health has been neglected by health and food policy makers. For example while there are specific government targets for smoking in England there are no equivalent dietary targets, the National Service Framework for Coronary Heart Disease ${ }^{23}$ has a specific standard for smoking cessation but no equivalent standard for dietary improvement.

The estimates could be improved by more sophisticated and systematic methods-for example by calculating appropriate PAFs and applying them to the burden of specific diseases rather than ICD chapters. The estimates should be refined, as without quantifying the burden of food related ill health we cannot say whether it is a problem worth worrying about or not.

\section{Authors' affiliations}

M Rayner, P Scarborough, British Heart Foundation Health Promotion Research Group, Department of Public Health, University of Oxford, UK

Funding: Mike Rayner and Peter Scarborough are funded by the British Heart Foundation.

Conflicts of interest: none declared.

\section{REFERENCES}

1 Murray CJL, Lopez AD. On the comparable quantification of the health risks: lessons from the blobal burden of disease study. Epidemiology 1999; 10:594-605.

2 Hollinghurst S, Bevan G, Bowie C. Estimating the 'avoidable' burden of disease by disability adjusted life years (DALYs). Health Care Management 2000;2:9-21.

3 World Health Organisation. Food and health in Europe: a new basis of action. Copenhagen: WHO Region Office for Europe, 2002.

4 World Health Organisation. Diet, nutrition and the prevention of chronic diseases. Report of a Joint WHO/FAO Expert Consultation. WHO Technical Report Series 916. Geneva: WHO, 2003.

5 World Health Organisation. World health report 2003. Geneva: WHO, 2003.

6 Arnesen T, Nord E. The value of the DALY life: problems with ethics and validity of disability adjusted life years. BMJ 1999;319:1423-5.

7 Murray CJL, Lopez AD. Global mortality, disability, and the contribution of risk factors: global burden of disease study. Lancet 1997;349:1436-42.

8 Ezzati M, Lopez AD, Rodgers A, et al. Comparative quantification of health risks. Geneva: WHO, 2004.

9 NHS Executive. Burdens of disease. A discussion document. Wetherby: Department of Health, 1996.

10 Department of Health. Choosing health: making healthier choices. London: DoH, 2004.

11 National Institute of Public Health, Stockholm. Determinants of the burden of disease in the European Union. Stockholm: NIPH, 1997.

12 Mathers CD, Vos ET, Stevenson CE, et al. The burden of disease and injury in Australia. Bull WHO 2001;79:1076-84.

13 European Heart Network. Diet, nutrition and cardiovascular disease prevention in the European Union. Brussels: EHN, 1998.

14 Doll R, Peto R. The cause of cancer: quantitative estimates of avoidable risks of cancer in the United States today. J Natl Cancer Inst 1981;66:1191-308.

15 World Cancer Research Fund. Food, nutrition and the prevention of cancer: a global perspective. Washington, DC: American Institute for Cancer Research, 1997.

16 Wanless D. Securing our future health: taking a long-term view. London: HM Treasury, 2002.

17 Department of Health. Department of Health resource accounts 2002-2003. London: The Stationery Office, 2004.

18 Yuen P. Compendium of health statistics: 2004. 16th ed. London: Office of Health Economics, 2005.

19 Petersen S, Peto V, Rayner M, et al. European cardiovascular disease statistics. London: British Heart Foundation, 2005.

20 Pretty JN, Brett C, Gee D, et al. An assessment of the total external costs of UK agriculture. Agricultural Systems 2000;65:113-36.

21 Parrott S, Godfrey C, Raw M, et al. Guidance for commissioners on the cost effectiveness of smoking cessation interventions. Thorax 1998;53(suppl 5):S2-37.

22 National Audit Office. Tackling obesity in England. London: The Stationery Office, 2001.

23 Department of Health. Coronary heart disease national service framework. London: The Stationery Office, 2000. 